\title{
The Effect of Clinical Pharmacists on Readmission Rates of Heart Failure Patients in the Accountable Care Environment
}

\author{
Christie Schumacher, PharmD, BCPS, BCACP; Golbarg Moaddab, MD; \\ Monique Colbert, APN; and Mary Ann Kliethermes, BS, PharmD, FAPhA
}

\begin{abstract}
BACKGROUND: Recent changes in the health care delivery landscape have expanded opportunities for clinical pharmacists in the ambulatory care setting. This article describes the successful integration of a clinical pharmacist-led chronic disease management service in a patient-centered medical home (PCMH) and accountable care organization (ACO) environment.

PROGRAM DESCRIPTION: In 2008, the year before PCMH implementation, $36 \%$ of patients who were hospitalized at Advocate Trinity Hospital for a heart failure exacerbation were readmitted within 30 days of their hospital stay for heart failure exacerbation. This high rate of heart failure hospital readmissions, compared with national standards, drove the implementation of the PCMH at Advocate Medical Group-Southeast Center (AMG-SE), the adjoining outpatient medical clinic. A clinical pharmacist was added to the health care team to help achieve the collective goal of improving patient outcomes and decreasing hospitalizations.
\end{abstract}

OBSERVATIONS: From November 1, 2009, through August 30, 2010, the clinical pharmacist conducted visits and intervened in the care of 111 chronic heart failure patients. A pre/post analysis of those 111 patients during the 10 months before and after the integration of the clinical pharmacist showed that those patients were hospitalized 63 times in the 10 months before having regularly scheduled visits with the clinical pharmacist and 30 times in the 10 months after establishing care. This reduction from 63 to 30 visits translated to an approximate $50 \%$ decrease in heart failure hospitalizations in patients being followed by the clinical pharmacist within the first 10 months. Once the clinical pharmacist became better integrated into the workflow through development of rapport with the medical team, the outcomes improved further. In an 18-month analysis from May 1, 2010, through November 30,2011 , only $2 \%$ of patients ( 3 of 153 ) designated as high-risk patients managed by the clinical pharmacist had a 30 -day readmission for heart failure exacerbation.

IMPLICATIONS: Outcomes-based models have expanded opportunities for clinical pharmacist involvement and can provide unique reimbursement options. Demonstration of cost savings and an improvement in quality measures are paramount to establishing and justifying the clinical pharmacist's role in a team-based model of care.

J Manag Care Spec Pharm. 2018;24(8):795-99

Copyright $\odot 2018$, Academy of Managed Care Pharmacy. All rights reserved. ccountable care organizations (ACOs) and patient$A$ centered medical homes (PCMHs) have emerged as 12 models of patient care that use an interprofessional team model to improve outcomes by focusing on comprehensive care and population health. Recent changes in the health care landscape have created a drive to improve quality while containing costs and, consequently, have created a demand for integrated delivery models. Financial incentives are made available to ACO providers for avoiding readmissions, preventable complications, and duplicate services, with incentives in the form of bonuses and shared savings. Overall, the ACO model holds providers accountable for the health of their patients, incentivizing them to work cooperatively and efficiently, and encouraging them to reduce costs by monitoring the care of their patients between and among providers. ${ }^{1-3}$

The ACO environment and outcomes-based reimbursement have created an opportunity for clinical pharmacists to pursue a larger role in the ambulatory care setting. ${ }^{4}$ Collaborative care models that include a clinical pharmacist have been shown to alleviate some of the demand for physician-provided care and facilitate access to primary care services, especially those related to medication monitoring and chronic disease management. ${ }^{5,6}$ Despite evidence that clinical pharmacists can improve patient outcomes through disease state management, many interdisciplinary models do not incorporate clinical pharmacists into the primary care team. ${ }^{5,6}$ This stems from lack of provider status, reimbursement models that rarely cover the cost of a clinical pharmacist, and lack of experience in how to embed pharmacists into a team-based care environment. ${ }^{3}$ Although some clinical pharmacists are becoming more involved in the primary care setting, understanding their value and optimal integration into the care team has yet to be described. With the increasing number of medications prescribed per patient, the need for chronic disease management, and the importance of medication adherence, there are many areas of opportunity for integration of clinical pharmacist services within the teambased care environment. ${ }^{3}$ This article describes the successful implementation of a clinical pharmacist-led chronic disease management service in the PCMH and ACO environment and the outcomes that were generated from its practice.

\section{Program Description}

The Advocate Medical Group-Southeast Center (AMG-SE), part of the AMG subsidiary of the Advocate Health System, is 
TABLE 1 Initial Data from Patients with DM and HF Managed by Clinical Pharmacist

Patients with HF Managed by Clinical Pharmacist, November 2009-August 2010

$(\mathbf{n}=111)$

Inpatient admissions for $111 \mathrm{HF}$ patients 10 months before PCMH implementation

Inpatient admissions for $111 \mathrm{HF}$ patients 10 months after PCMH implementation

Average cost per HF admission

Potential cost avoidance for $111 \mathrm{HF}$ patients based on pre/post enrollment

Patients with DM Managed by Clinical Pharmacist, May 2010-November 2011

\begin{tabular}{|c|c|c|c|}
\hline & Baseline Value & Study Period Conclusion & Change \\
\hline Average Alc (\%) & $9.2 \quad( \pm 2.6)$ & $7.7 \quad( \pm 1.7)$ & -1.5 \\
\hline Number of patients with Alc $<7 \%^{a}$ & $25 \quad(16 \%)$ & $49 \quad(32 \%)$ & $24 \quad(16 \%)$ \\
\hline Average LDL (mg/dL) & $97 \quad( \pm 30.6)$ & $81 \quad( \pm 25.6)$ & -16 \\
\hline Patients with an $\mathrm{LDL}<100 \mathrm{mg} / \mathrm{dL}$ & $81 \quad(53 \%)$ & $103 \quad(67 \%)$ & $22 \quad(14 \%)$ \\
\hline Average systolic blood pressure (mmHg) & $143( \pm 22.1)$ & $129 \quad( \pm 17.9)$ & -14 \\
\hline Average diastolic blood pressure $(\mathrm{mmHg})$ & $77 \quad( \pm 10.6)$ & $70 \quad( \pm 10.8)$ & -7 \\
\hline Patients on ACEI or ARB & $110 \quad(72 \%)$ & $133 \quad(87 \%)$ & $23 \quad(15 \%)$ \\
\hline Patients taking statin & $92 \quad(60 \%)$ & $121 \quad(79 \%)$ & $29 \quad(19 \%)$ \\
\hline
\end{tabular}

${ }^{a}$ Before American Diabetes Association guidelines created less stringent goals.

$A l c=$ hemoglobin Alc; $A C E I=$ angiotensin-converting enzyme inhibitor; $A R B=$ angiotensin receptor blocker; $D M=$ diabetes mellitus; $H F=$ heart failure; $L D L=$ low-density lipoprotein; $P C M H=$ patient-centered medical home.

located in a lower-income neighborhood in Chicago, Illinois The population is composed predominantly of African American patients, with a small percentage of Hispanic patients. Seventy percent of patients at the site are under a full-risk global payment model through Medicare Advantage or commercial payers, and 20\% are under a shared savings program. This payment structure incentivizes the center to delegate care to the most appropriate team member based on their scope of practice to keep patients well and costs low. With the focus shifting toward preventive health care, the goal is to meet performance measures, prevent hospitalizations, and decrease costs. This is best accomplished through the team-based model of care. The ACO model, which offers incentives by providing an increase in reimbursement for an improvement in outcomes, promotes the value of any health care provider that can demonstrate a positive impact on patient outcomes and cost savings. These new models of care offer an opportunity for clinical pharmacists to demonstrate their value as a team member in providing patient care.

In 2008, AMG created a PCMH project team to improve outcomes at AMG-SE. At the time, AMG-SE had the highest rate of heart failure hospitalizations and 30-day readmissions among more than 250 AMG centers in the Chicago metropolitan area. In the year before PCMH implementation, 36\% of patients who were hospitalized at Advocate Trinity Hospital for a heart failure exacerbation were readmitted within 30 days of their hospital stay for a subsequent heart failure exacerbation. Over $50 \%$ of patients hospitalized for heart failure during this period had a primary care physician at AMG-SE. A comprehensive care structure did not exist and there were minimal coordinated patient handoffs both among providers within
AMG-SE and between the center and the adjoining Advocate Trinity Hospital. A high rate of heart failure hospital readmissions and poorly controlled chronic disease states led to a desire to implement the PCMH model at the center to achieve the collective goal of improving the quality of patient care and decreasing hospitalizations. The initial goal of the PCMH pilot was to focus on improving the care of patients diagnosed with chronic heart failure.

A clinical pharmacist was hired in 2009 through a shared contract with a college of pharmacy and had previous postgraduate year 1 pharmacy practice residency training and an additional heart failure traineeship. The clinical pharmacist was initially appointed to the clinical operations and analytics teams of the PCMH pilot, which was scheduled to be implemented in November 2009. The initial focus of the clinical pharmacist was to improve hospitalization rates and 30-day readmission rates for heart failure through more frequent follow-up visits and improved access to care. Clinic space including a patient visit room and an independent provider schedule were established for the clinical pharmacist. Although the initial focus was on the care of patients with heart failure, the population needs for other comorbid chronic conditions quickly became evident, creating a need to expand services and the role of the clinical pharmacist within months of establishing the practice.

\section{Observations}

\section{Building the Interprofessional Model and Expanding the Pharmacist Role}

The AMG-SE PCMH was created as an interdisciplinary team including 6 primary care physicians, an advance practice nurse 
The Effect of Clinical Pharmacists on Readmission Rates of Heart Failure Patients in the Accountable Care Environment

TABLE 2 AMG-SE Quality Metrics Dashboard Demonstrating Trends in Chronic Disease Outcomes After PCMH Initiation Starting in 2010

\begin{tabular}{|c|c|c|c|c|c|c|}
\hline & 2009 Results ${ }^{a}$ & 2010 Results & 2011 Results & 2012 Results & 2013 Results & 2014 Results \\
\hline \multicolumn{7}{|l|}{ Diabetes management, aged $\geq 65$ years, $\%$} \\
\hline Annual eye exam & 35 & 67 & 70 & 69 & 71 & 75 \\
\hline Annual foot exam & 71 & 96 & 96 & 92 & 86 & 84 \\
\hline Alc performed & 80 & 95 & 95 & 93 & 93 & 96 \\
\hline Alc performed $<8 \%$ & 32 & 45 & 64 & 66 & 84 & 80 \\
\hline Alc performed $>9 \%$ & 41 & 29 & 23 & 21 & 9 & 11 \\
\hline LDL performed & 78 & 94 & 59 & 92 & 94 & 93 \\
\hline $\mathrm{LDL}<100 \mathrm{mg} / \mathrm{dL}$ & 40 & 55 & 59 & 57 & 63 & 65 \\
\hline $\mathrm{LDL}>130 \mathrm{mg} / \mathrm{dL}$ & 39 & 26 & 17 & 20 & 19 & 16 \\
\hline Hypertension control $(<140 / 90)$ & 62 & 82 & 84 & 91 & 91 & 91 \\
\hline Nephropathy testing & 85 & 75 & 92 & 94 & 98 & 100 \\
\hline \multicolumn{7}{|l|}{ Generic medication use, \% } \\
\hline Generic medication use & 80 & 84 & 86 & 89 & 92 & 92 \\
\hline Generic statins use & 75 & 87 & 82 & 91 & 96 & 95 \\
\hline \multicolumn{7}{|l|}{ HF management, \% } \\
\hline Appropriate medication: ACEI or ARB in HF & 74 & 94 & 91 & 100 & 88 & 95 \\
\hline Appropriate medication: BB in HF & 82 & 84 & 91 & 100 & 80 & 83 \\
\hline
\end{tabular}

a Before the clinical pharmacist managed patients at the center.

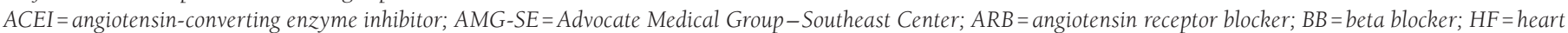
failure; $L D L=$ low-density lipoprotein; $P C M H=$ patient-centered medical home.

(APN), the clinical pharmacist, a dietician, a nurse, 3 patient care managers, and a physician assistant responsible for acute care appointments. In the initial implementation stages, the clinical pharmacist worked with the AMG-SE cardiologist and was able to demonstrate competence and gain the cardiologist's trust and support for heart failure medication management during specialist visits. The clinical pharmacist provided comprehensive medication management services with interventions that included initiating and titrating guideline-directed medical therapy (GDMT), assessing patients' reported symptoms and clinical status, adjusting diuretic therapy appropriately, identifying and removing any medications that may exacerbate heart failure, and educating patients on self-management and monitoring techniques (e.g., daily weight monitoring, sodium and fluid restriction as indicated, and home blood pressure monitoring). ${ }^{7}$ The clinical pharmacist also regularly monitored for barriers to adherence, such as costs of care and patient understanding of therapy importance. The clinical pharmacist's effect on the primary goal of improving the care of patients with heart failure was measured by hospitalizations and hospital readmissions for heart failure. Additional goals were to meet the HEDIS and CMS star measure metrics that the organization was responsible for based on value-based payment contracts with federal and commercial payers.

During the visits for most of the heart failure patients, the clinical pharmacist identified the need for improvement in the comprehensive management of other comorbidities, such as diabetes mellitus (DM), dyslipidemia, hypertension, thyroid disorders, gout, and chronic obstructive pulmonary disease (COPD) and/or asthma. The need to expand the collaborative practice was presented to the physicians.

Expansion of the clinical pharmacist's role beyond heart failure collaborative management began with patients with DM due to the high percentage of patients with DM at AMG-SE and lack of an endocrinologist on the multidisciplinary team. A review of outcomes data tracked by the clinical pharmacist (Table 1) and data from the AMG-SE clinical dashboard (Table 2) indicated that the care of patients with DM was improving as providers remained stable during this time frame, and the implementation of diabetes management by the clinical pharmacist was the only intervention. The clinical pharmacist was subsequently charged with further defining and expanding the role of the clinical pharmacist in the PCMH and the development of resources. This included writing and implementing best practice protocols for heart failure, diabetes, asthma, COPD, hypertension, and hyperlipidemia.

\section{AMG-SE Outcomes}

Outcomes from the first 10 months of implementation at AMG-SE are presented in Table 1. From November 1, 2009, to August 30, 2010, the clinical pharmacist intervened in the care of 111 patients with chronic heart failure. A pre/post analysis of those 111 patients during the 10 months before and after the integration of the clinical pharmacist on November 1, 2009, showed that the 111 patients were hospitalized for heart failure 63 times in the 10 months before regularly scheduled visits 
with the clinical pharmacist and 30 times in the 10 months after establishing care from November 2009 to August 2010. These data were tracked and analyzed by the PCMH operations and analytics team at AMG-SE using insurance claims and were confirmed through an internal analysis that used the hospital census and patient-reported hospitalizations during visits.

The decrease from 63 hospitalizations in the patient population before management by the clinical pharmacist to 30 hospitalizations translated to an approximate 50\% decrease in hospitalizations in patients being followed by the clinical pharmacist within the first 10 months. The main intervention that changed in the 10-month period that led to the decrease in hospitalization was the clinical pharmacist, who provided comprehensive medication management that included initiating and titrating GDMT, assessing patients' reported symptoms and clinical status, adjusting diuretic therapy appropriately, identifying and removing any medications that may exacerbate heart failure, and educating patients on self-management and monitoring techniques.

From an internal cost analysis, based on the average heart failure hospitalization cost at Advocate Trinity Hospital of $\$ 8,500$, a potential cost avoidance of $\$ 280,000$ was determined. This cost was calculated by using the difference in hospitalizations before and after the clinical pharmacist intervention in the 111 patients, which was 33, and multiplying that by the average cost of each hospitalization for heart failure, $\$ 8,500$.

From May 1, 2010, to November 30, 2011, the clinical pharmacist's panel increased to 153 patients, and additional outcomes data were collected. Over an 18-month period starting in May 2010, only 3 of the 153 AMG-designated high-risk patients managed by the clinical pharmacist for heart failure had a 30-day readmission for a heart failure exacerbation. Patients were identified as high risk if they had been hospitalized in the previous year for heart failure based on medical claims reports or were deemed high risk and referred by their cardiologist or primary care physician if they had been hospitalized or had an ejection fraction less than 35\%.

Patients with DM were also referred to the clinical pharmacist for management if their hemoglobin Alc level was greater than $9 \%$ or if they were hospitalized for hyperglycemia or a complication of DM. Initial outcomes for patients with DM managed by the clinical pharmacist were collected (Table 1). The improved outcomes for patients with DM were the primary driver for the physicians' approval of expansion of the clinical pharmacist's collaborative role in the care of other chronic disease states at AMG-SE.

Expansion of the clinical pharmacist's role to include care for patients with DM resulted in the recognition of AMG-SE as 1 of the top 5 AMG sites for diabetes management in 2012, out of approximately 250 AMG sites in the Chicago metropolitan area. The recognition was based on demonstrated improvement in AMG diabetes quality metric measurements, such as the percentage of patients receiving an annual eye exam, annual foot exam, or annual nephropathy screening; Alc performed; patients with a Alc less than $8 \%$ and greater than $9 \%$; and other disease state measures, including blood pressure control and cholesterol management (Table 2). In addition to the clinical pharmacist managing patients with uncontrolled diabetes, clinical discussions and recommendations to physicians facilitated the improvement in disease state management at the site.

Quality metrics that improved after the integration of the clinical pharmacist included generic medication use and the number of patients on an angiotensin-converting enzyme inhibitor and a beta-blocker for heart failure management (Table 2). The initial results of the PCMH pilot program led to the justification for and employment of more clinical pharmacists at AMG-SE and other AMG ambulatory care sites.

\section{Implementation Challenges}

There were 2 significant initial challenges in integrating the clinical pharmacist into the AMG-SE PCMH: (1) lack of familiarity of the clinical pharmacist's role, education, and skill set by the physicians at AMG-SE; and (2) limited exposure to a clinical pharmacist working as part of the collaborative health care team. Before PCMH implementation, AMG only used clinical pharmacists for anticoagulation services and did not have clinical pharmacists involved in chronic disease management. At the time, models at different AMG centers used an APN to provide heart failure and chronic disease management due to physician comfort level through experience with APNs as providers, as well as their ability to bill for services and prescriptive authority.

At AMG-SE, it was challenging for physicians to understand the concept of prescribing privileges for the clinical pharmacist when it was introduced at meetings by the $\mathrm{PCMH}$ project team. The physicians expressed concern regarding the clinical pharmacist's lack of direct prescriptive authority and the associated liability of the clinical pharmacist prescribing under their name. Ultimately, their resistance to the clinical pharmacist and lack of experience with team-based care decreased over time as the physicians worked more closely with the clinical pharmacist. Because the initial focus was on the care of heart failure patients and the improvement in clinical outcomes, the experience and competency of the clinical pharmacist were validated, which led to increased physician confidence and the further expansion of clinical pharmacy services to other disease states.

In the ACO model, physicians are incentivized based on outcomes and are therefore more likely to include other members of the health care team (e.g., clinical pharmacist, APN, or physician assistant) to assist in generating the best outcomes for their patients. By demonstrating improvement in clinical outcomes, the clinical pharmacist was able increase the scope of practice and patient panels through physician referrals. 
A second influential factor to gain physician support of the clinical pharmacist to assist in the management of chronic disease states was through educational presentations at physician meetings. Through these presentations, which included recommendations supported by the primary literature and guidelines, physicians gained a better understanding of the extensive knowledge base of a clinical pharmacist and became more comfortable with the clinical pharmacist prescribing under their names.

A major challenge arose during the expansion process as clinical pharmacy services were introduced at other AMG centers. Not all centers used the same workflow and staffing models, and variability in skill sets existed among clinical pharmacists. This required clinic-specific discussions about the needs of the site and where the clinical pharmacist could best fill gaps in primary care to improve patient outcomes. This variability in clinical experience and skill sets demonstrated how critical it was to find the right person for the right site.

\section{Implications}

Recent changes in the health care delivery landscape have opened new doors for clinical pharmacists to provide more comprehensive disease state management in the ambulatory care setting. As clinical pharmacists continue to expand their role in ambulatory care, a strong working relationship with physicians and other providers, such as APNs and physician assistants, is critical to success. While clinical pharmacists continue to develop their role in team-based care, it is important to demonstrate the value of clinical pharmacy services and competency in chronic disease management in the ambulatory care setting. By initially focusing on the management of 1 disease state and gathering outcomes data, the clinical pharmacist at AMG-SE could garner trust with physicians and expand their collaborative role in the service to include other chronic disease states.

The experience at AMG-SE has shown the importance of choosing a disease state where clinical outcomes can be demonstrated and measured in a timely manner. The improvement of outcomes, such as decreased hospitalizations and emergency department visits, in costly disease states (e.g., COPD and heart failure) can demonstrate the value of clinical pharmacy through cost savings. Once cost savings have been demonstrated and the role of the clinical pharmacist has been initiated, the responsibilities of the clinical pharmacist can be expanded to other chronic disease states.

A key to success at AMG-SE was having a clinical pharmacist in an administrative position with a lead role on the PCMH operations and analytics teams. Strong leadership and continued support to ensure the clinical pharmacist grew professionally were key in the successful integration of the clinical pharmacist at this site. Other skills that facilitated implementation included strong communication skills and the ability to work with a population that was challenging due to low health literacy and socioeconomic barriers. A strong knowledge base and ability to support recommendations with the most recent primary literature and guidelines were cited by physicians as the most important factors when granting and expanding clinical pharmacist prescribing. The physicians in the center felt more comfortable allowing the clinical pharmacist to prescribe under their names for a variety of different disease states when the clinical pharmacist could provide evidence-based recommendations by describing updates in guidelines and was comfortable discussing primary literature application.

With the implementation of ACOs and quality-based incentives, clinical pharmacists are able to demonstrate their value as part of an interdisciplinary health care team. Outcomes-based models have provided opportunities for increased clinical pharmacist involvement and may provide unique reimbursement opportunities in team-based models of care.

\section{Authors}

CHRISTIE SCHUMACHER, PharmD, BCPS, BCACP, Midwestern University Chicago College of Pharmacy, Downers Grove, Illinois, and Advocate Medical Group, Southeast Center, Chicago, Illinois. GOLBARG MOADDAB, MD, Advocate Medical Group, Irving and Western Center, Chicago, Illinois; MONIQUE COLBERT, APN, Rush University Medical Center, Chicago, Illinois; and MARY ANN KLIETHERMES, BS, PharmD, FAPhA, Midwestern University Chicago College of Pharmacy, Downers Grove, Illinois.

AUTHOR CORRESPONDENCE: Christie Schumacher, PharmD, BCPS, BCACP, Associate Professor, Pharmacy Practice, Midwestern University Chicago College of Pharmacy, 555 31st St., Downers Grove, IL 60515. Tel.: 773.356.8241; E-mail: cschum@midwestern.edu.

\section{DISCLOSURES}

No outside funding supported this research. The authors have no conflicts of interest to disclose.

\section{REFERENCES}

1. Nester J. The importance of interprofessional practice and education in the era of accountable care. N C Med J. 2016;77(2):128-32.

2. Kellerman R, Kirk L. Principles of the patient-centered medical home. Am Fam Physician. 2007;76(6):774-75.

3. Rosenthal TC. The medical home: growing evidence to support a new approach to primary care. J Am Board Fam Med. 2008;21(5)427-40.

4. Joseph T, Hale GM, Eltaki SM, et al. Integration strategies of pharmacists in primary care-based accountable care organizations: a report from the accountable care organization research network, services, and education. J Manag Care Spec Pharm. 2017;23(5):541-48. Available at: https://www.jmcp. org/doi/10.18553/jmcp.2017.23.5.541.

5. Helling DK, Johnson SG. Defining and advancing ambulatory care pharmacy practice: it is time to lengthen our stride. Am J Health Syst Pharm. 2014;71(16):1348-56.

6. Smith M, Bates DW, Bodenheimer TS. Pharmacists belong in accountable care organizations and integrated care teams. Health Aff (Millwood) 2013;32(11):1963-70.

7. American College of Clinical Pharmacy. Standards of practice for clinical pharmacists. Pharmacotherapy. 2014;34(8):794-97. 